
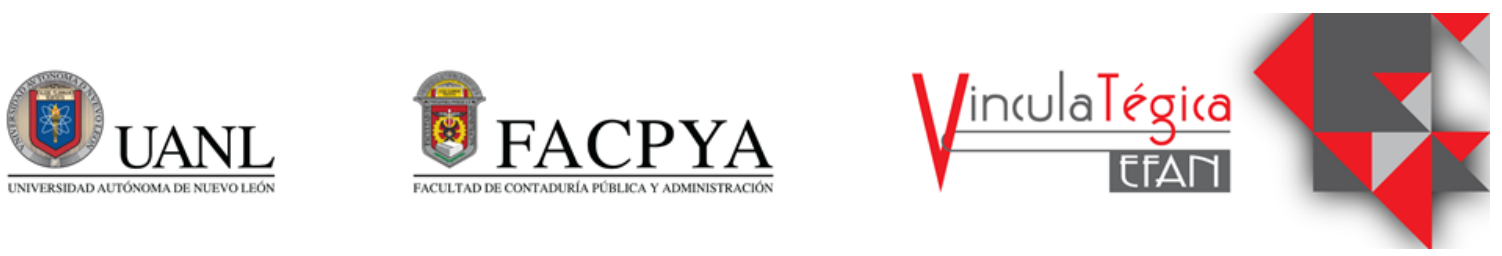

\title{
Factores que influyen en la intención de revisitar los restaurantes en un contexto de pandemia
}

\author{
Christian Reich López ${ }^{1}$ y María Mayela Terán Cázarez ${ }^{2}$ \\ ${ }^{1}$ Universidad Autónoma de Nuevo León, Facultad de Contaduría Pública y Administración Monterrey, \\ Nuevo León, México, creichl@uanl.edu.mx,Av.UniversidadS/N \\ Col. Ciudad Universitaria, (+52) 8183204080 \\ ${ }^{2}$ Universidad Autónoma de Nuevo León, Facultad de Contaduría Pública y Administración Monterrey, \\ Nuevo León, México,mayela.teran@gmail.com \\ Av. Universidad $S / N$ \\ Col. Ciudad Universitaria, (+52) 8183294080
}

Información del artículo revisado por pares

Fecha de aceptación: junio-2021

Fecha de publicación en línea: diciembre-2021

DOI: https://doi.org/10.29105/vtga7.2-62

\section{Resumen}

El objetivo de esta investigación fue analizar las variables que inciden en la intención de revisitar un restaurante de servicio completo en México en el contexto de la pandemia por COVID-19. El método para la estimación del modelo fue a través de regresión lineal múltiple. Los resultados indican que la intención de revisitar depende de la calidad de la comida, el valor percibido por el cliente, la calidad de la interacción del personal y la confianza. La originalidad del artículo consiste en la aportación del entendimiento del consumidor en un contexto de reapertura en la pandemia, destacando que la confianza obtuvo una beta mayor que el resto de las variables, hallazgo que puede ser explicado a través del Modelo de Creencias de la Salud.

Palabras clave: COVID-19, Intención de revisita, México, restaurantes, pandemia.

\section{INTRODUCCIÓN}

El año 2020 ha sido un momento histórico para la industria de la hospitalidad, la cual a causa de la pandemia mundial de COVID-19 ha experimentado una crisis sin precedente de escala mundial. Sin duda alguna, el nicho restaurantero ha sido de los más afectados,
Abstract

The objective of this research was to analyze the variables that are related to the revisit intention of fullservice type restaurant in Mexico in the context of the COVID-19 pandemic. The chosen method was a multiple regression. The results show evidence that the re-visit intention in a pandemic context depends on quality of the food, perceived value, personal interaction quality and trust. The originality of the article resides in the contribution about consumer behavior in a pandemic re-opening context, highlighting that trust scored a higher beta than the rest of the variables, finding that may be explained by the Health Belief Model.

Keywords: COVID-19, re-visit intention, Mexico, restaurants, pandemic.

JEL: D11, D12

debido a esta situación. Statista (2021) proyecta que la recuperación global de la hospitalidad recuperará niveles de 2019 hasta el año 2025 (ver figura 1). Específicamente sobre el entorno restaurantero, los autores Severson \& Yaffe-Bellany (2020) afirman que "más de la mitad de los restaurantes no 
sobrevivirán a esta situación” (pág. 21).

La industria restaurantera en México constituye un pilar importante en la economía del país, acorde a los censos económicos del 2014 llevados acabo por el Instituto Nacional de Estadística y Geografía o INEGI, esta industria constituye una participación de alrededor del $1.8 \%$ del PIB, involucrando cerca de 500 mil establecimientos y generando $6.8 \%$ del total de los empleos del país.

Dejando de lado la pandemia, el sector restaurantero ha sido históricamente reconocido como uno de los más duros en relación con la tasa de sobrevivencia de negocio.

Los autores Parsa, Self, Njite, \& King (2005) identificaron que el $61.36 \%$ de los restaurantes operados de forma independiente quiebran en los primeros 3 años de existir, la alta complejidad del ramo, aunado al gran reto que representa una cuarentena en un contexto de pandemia, ha hecho que muchos negocios de este giro bajen la cortina, y que los que se encuentran operando se encuentren en modo de supervivencia (págs. 304-322).

En ese sentido, el problema de investigación al cual apunta este estudio es conocer cuales son las variables que influyen en la intención de volver a visitar un restaurante en un contexto de reapertura de pandemia, de esta forma se busca contribuir en el entendimiento del comportamiento de los consumidores en tiempos de crisis sanitarias. El buscar dicho entendimiento puede beneficiar a los practicantes de la industria para tomar las medidas adecuadas que potencialmente pueden impactar de forma positiva en la satisfacción de sus clientes y por consecuencia en las ventas.

$\mathrm{Al}$ momento del levantamiento de los datos para esta investigación, la industria se encontraba con restricciones preventivas por parte del gobierno, como por ejemplo operar con un aforo del $30 \%$ de la capacidad total, de tal forma que los casos seleccionados para el análisis estadístico de este documento cumplen con la característica de ser comensales los cuales salieron a consumir al menos una vez a algún restaurante del tipo de servicio completo durante estas restricciones sanitarias, tomando en consideración que al tener medidas especiales de operación, la experiencia de recibir un servicio de este tipo cambia al tener influencia de un contexto especial.

\section{MARCO TEÓRICO}

Los restaurantes al pertenecer a una industria que resulta relevante para la mayoría de las economías de diversos países han sido unidades de estudio para varios investigadores interesados en comprender de mejor manera el comportamiento de los clientes, así como las variables que influyen en diversos comportamientos y actitudes como pueden ser la lealtad, la recomendación de boca en boca o la intención de revisitar de los comensales. La tabla 1 muestra un breve compendio de algunos estudios empíricos realizados en esta industria, así como las variables involucradas, el método y el área geográfica donde se realizaron las investigaciones.

\subsection{Modelos de salud}

En relación con el comportamiento de los consumidores, se han desarrollado a lo largo del tiempo diversas teorías que buscan generar marcos conceptuales en circunstancias tales como crisis, pandemias o tiempos de cambio. En el caso de crisis sanitarias Murray \& Schaller (2010), afirman que "el comportamiento cambia debido al miedo que las personas tienen al percibir la amenaza de una enfermedad potencial, de tal manera que también se alteran las formas en que un cliente consume" (págs. 387-409).

El marco conceptual para este trabajo de investigación es denominado Modelo de Creencias de la Salud o Health Belief Model. "Este modelo propone que las actividades que tienen relación con la salud están en función de 3 tipos de factores o creencias" (Maiman \& Becker, 1974, págs. 336-353). En primer lugar, los factores modificables, en los cuales se encuentran las características demográficas del sujeto de estudio. La combinación de factores como son género, edad, ubicación geográfica, grupo étnico, conocimiento de salud, ingreso, entre otros marcarán precedente en el segundo tipo de factor el cual son las creencias individuales. En el contexto 
de una pandemia, estas creencias son principalmente el reconocer que tan susceptible se es a la enfermedad, que beneficios percibe el consumidor de tomar una acción preventiva ante un potencial contagio y en caso de contagiarse, el evaluar que tan severo puede ser el caso. Finalmente, el tercer factor recae en la acción o comportamiento el cual deriva de los dos factores anteriores, en este contexto la acción apunta a decisiones como salir a consumir a un restaurante o en este caso decidir volver a visitar el mismo restaurante en el futuro.

\subsection{Calidad de la comida}

La calidad de la comida (CQ) ha sido reportada en diversos estudios como la dimensión más importante que antecede comportamientos y actitudes como la satisfacción, la lealtad o la recomendación de boca en boca en el contexto restaurantero" (Mattila, 2001, págs. 73-79). A pesar de que es una variable bastante estudiada, no se ha estandarizado una definición del constructo. Para los fines de esta investigación, se propone el uso de las dimensiones de Namkung \& Jang (2007), las cuales consisten en la atractividad visual de los platillos, que estos sean servidos en la temperatura adecuada y que posean buen sabor a percepción del comensal.

Partiendo de la revisión de la literatura, se establece la primera hipótesis de investigación.

H1: La calidad de la comida (CQ) influye de forma directa y positiva en la intención de revisitar (IR) por parte del cliente en un contexto de pandemia.

\subsection{Valor percibido por el cliente}

El valor percibido por el cliente (VPC) consiste en la evaluación del cliente entre la expectativa del servicio, la percepción real que experimentó y el precio pagado por el mismo" (Sweeney \& Soutar, 2001, págs. 203-220). Los autores Zeithaml, Parasuraman, Berry, \& Berry (1990) reconocen en sus investigaciones que existe un balance por parte del cliente entre la expectativa del servicio y la percepción del mismo, en caso de que este balance sea positivo, es decir que la percepción del servicio recibido sea mayor que la expectativa, generará satisfacción en el cliente, el cual se ha estudiado es un estado que antecede diversos comportamientos benéficos para un restaurante como generar recompras en el futuro. Debido a lo anterior, se considera una variable relevante de la cual se desprende la segunda hipótesis.

H2: El valor percibido por el cliente (VPC) influye de forma directa y positiva en la intención de revisitar (IR) por parte del cliente en un contexto de pandemia.

\subsection{Calidad de la interacción del personal}

La calidad de la interacción del personal (CIP) es definida por Vesel \& Zabkar (2010) como "la habilidad del proveedor de generar, mantener y desarrollar una interacción positiva y abierta con sus clientes con el fin de establecer conexiones a largo plazo".

Según Harker \& Egan (2006) esta variable se desprende de la teoría del marketing relacional, finalmente el personal de primera línea es el que se percibe en el entorno restaurantero de ser el portavoz del servicio al cliente, aunque exista un equipo operativo detrás para poder lograr que todo funcione tal y como lo espera un cliente (págs. 215-242).

Debido a estos antecedentes es que se desprende la tercera hipótesis de estudio.

H3: La calidad de la interacción del personal (CIP) influye de forma directa y positiva en la intención de revisitar (IR) por parte del cliente en un contexto de pandemia.

\subsection{Confianza}

En un contexto de crisis sanitaria, la confianza (CF) es definida por Ling, Chai, \& Piew (2010) como "la expectativa del comportamiento, atribuible a una persona, objeto, producto, organización, empresa, institución de gobierno o cualquier otro que tenga un rol profesional" (págs. 1-26). "En el estudio de la comunicación, la confianza (CF) ha sido un constructo clave para su investigación, sin embargo, ha existido controversia en las dimensiones asociadas a su medición" (Freimuth, Musa, Hilyard, Quinn , \& Kim, 2014, págs. 321-339). En un contexto de pandemia, esta variable ha sido investigada, 
sin embargo, existen brechas pues el enfoque que se le ha dado a los estudios es mayormente evaluando a las instituciones que generan políticas públicas y no a los negocios en su comunicación o prácticas. Considerando lo anterior, así como el modelo de creencias de la salud, se propone la cuarta hipótesis.

$\mathrm{H} 4$ : La confianza (CF) influye de forma directa y positiva en la intención de revisitar (IR) por parte del cliente en un contexto de pandemia.

\subsection{Intención de revisitar}

La variable dependiente en este estudio es la intención de revisitar (IR). La definición por parte de Chien, Hong, \& Guo (2017) es la "predisposición que tiene un cliente de volver a visitar el mismo lugar" (págs. 552-561). Es por esta razón que en un contexto de pandemia esta intención se evalúe y se logren identificar los factores que generan el efecto. Los autores Rajput \& Gahfoor (2020) consideran que esta actitud se encuentra en el centro y corazón del compromiso y de la lealtad del cliente (págs. 1-12). Por lo anterior se propone intención de revisitar como variable dependiente en esta investigación.

\section{MÉTODO}

El diseño de esta investigación es cuantitativa, explicativa, exploratoria, no experimental y de corte transversal, considerando la teoría de los modelos de la salud como marco conceptual. La recolección de información fue a través de una encuesta auto administrada la cual se creó en el software kindorse surveys. Esta encuesta se distribuyó a través de redes sociales usando un tipo de muestreo no probabilístico por conveniencia, la metodología para hacer el contraste de hipótesis fue a través de la regresión lineal múltiple.

\subsection{Diseño del instrumento.}

El instrumento fue construido con base en la revisión de la literatura para poder adaptar items previamente validados. La variable calidad de los alimentos fueron adaptados de Namkung \& Jang, (2008), el valor percibido por el cliente se adaptó de Chen (2012), la calidad de la interacción del personal fue adaptado de Vesel \& Zabkar (2009), la confianza fue adaptado de Kim, Ferrin, \& Rao (2008) y la intención de revisitar fue adaptado de Chen, Htaik, Hiele, \& Chen (2017). Todos los reactivos fueron evaluados en una escala likert de 5 puntos en formato smiley. 3.2

\subsection{Muestra.}

El levantamiento de los datos se llevó acabo entre los días 9 y 17 de julio de 2020. El total de respuestas recibidas fue de 472 casos, sin embargo, solo se consideraron válidos solo 323. Se siguieron 3 criterios para considerar válida una respuesta y de tal forma llegar a la selección de los casos: 1) Haber contestado de forma positiva la pregunta filtro $¿$ Ha visitado algún restaurante en sus instalaciones en los últimos 60 días?, esto con el fin de asegurarnos de que el último consumo fue en un periodo de restricción por pandemia, 2) El restaurante evaluado o recordado para contestar la encuesta debió tener características de servicio completo (el comensal debe ser atendido sentado en una mesa por un mesero) y 3) Se eliminaron los casos cuyos patrones fueron repetidos para evitar un potencial sesgo por respuesta. En la tabla 3 se pueden observar los demográficos de la muestra.

\section{RESULTADOS}

\subsection{Análisis de fiabilidad}

La consistencia y fiabilidad de los items se midieron a través del alfa de Cronbach, obteniendo buen resultado de los elementos que integran las variables. Los resultados obtenidos fluctuaron entre 0.70 y .907 , como se puede observar en la tabla 4. Acorde a Mallery \& George (2000) los valores de alfa de cronbach mayores a 0.70 son considerados satisfactorios para investigaciones de tipo exploratorio. Todos los resultados obtenidos en esta investigación fueron procesados a través del software SPSS versión 25.

\subsection{KMO, prueba de Bartlett, $y$ comunalidades.}

Acorde a Hair Jr., Sarstedt, Hopkins, \& Kuppelwieser (2014) el principal objetivo de realizar análisis factorial es poder 
condensar la información de las variables originales en un número mas pequeño de items sin comprometer perdida de información.

En esta investigación se realizó un análisis factorial confirmatoria (CFA) obteniendo como resultado una prueba de esfericidad de Bartlet significativa, como se puede apreciar en la tabla 5. De igual manera todos los factores cargaron en el componente principal correspondiente con valores por encima de 0.70 (véase tabla 6), lo cual es evidencia suficiente para concluir que existe consistencia estructural en las variables.

\subsection{Estadística descriptiva: media, desviación estándar y correlación de Pearson.}

Una vez que se validó el instrumento a través del análisis factorial y del alfa de cronbach, se procedió a hacer el análisis descriptivo, en la tabla 7 se puede apreciar la media, desviación estándar y correlación de Pearson de las variables independientes, de las cuales se concluye que todas las correlaciones son significativas entre si con un p-valor > 0.000 .

\subsection{Regresión lineal múltiple}

Posterior al análisis de fiabilidad y de calcular los descriptivos de la muestra se procedió a generar una regresión múltiple acorde a las hipótesis planteadas en la investigación. Esta regresión arrojó una $\mathrm{R}$ cuadrada de 0.432 , lo que indica que el ajuste lineal del modelo es aceptable y que las variables independientes presentan el $43.2 \%$ de la varianza en la variable de respuesta o independiente.

El coeficiente de Durbin-Watson fue obtenido de igual manera, teniendo un valor de 1.9, con el cual la independencia entre los residuos de la regresión se puede asumir y por lo tanto concluir que no existe auto correlación, esta conclusión se obtiene cuando el coeficiente tiene un valor mayor a $1.5 \mathrm{y}$ menor a 2.5. Véase tabla 8 .

La tabla 9 muestra el análisis de ANOVA de la regresión en el cual se puede concluir que el modelo propuesto es significativo con un p-valor $<0.000$.
Los coeficientes del modelo de la regresión pueden ser apreciados en la tabla 10. Al haberse realizado la regresión por el método stepwise, las variables presentadas en el modelo son significativas con $\mathrm{p}$-valor $<0.05$ y p-valor $<0.000$. De igual manera el valor de inflación de la varianza (VIF) descarta problemas de multicolinealidad al presentar valores menores a 3 .

Con los valores previamente presentados, se construye la siguiente ecuación de regresión del modelo:

$$
\begin{gathered}
Y=\beta_{0}+\beta_{1} X_{1}+\beta_{2} X_{2}+\beta_{3} X_{3}+\beta_{4} X_{4}+E \\
Y=0.233+0.193 X_{1}+0.234 X_{2}+0.168 X_{3}+0.317 X_{4}+E
\end{gathered}
$$

\subsection{Contraste de hipótesis.}

En la tabla 11 se pueden apreciar las hipótesis presentadas y los resultados que se concluyen.

\section{CONCLUSIONES}

La evidencia en esta investigación permite concluir que la calidad de la comida, el valor percibido por el cliente, la calidad de la interacción del personal y la confianza son factores que influyen en la intención de un cliente de volver a visitar nuevamente un restaurante, estos hallazgos son consistentes con otros autores que han estudiado estas variables (Rajput \& Gahfoor (2020); Sivadas \& Jindal, (2017); Jalilvand, Salimipour, Elyasi, \& Mohammadi (2017); Adam, Adongo, \& Dayour (2015)). Sin embargo, lo que resulta particularmente interesante en este estudio es el contexto de pandemia, lo cual genera un efecto no observado de forma previa en otros estudios. De manera consistente, la calidad de la comida suele ser la variable que mayor influencia tiene en el efecto estudiado en el entorno restaurantero, esto ha sido analizando la satisfacción, la intención de recomendación y también en la intención de volver a visitar un negocio como variables independientes, por el contrario, en esta ocasión el peso de las variables fue diferente.

En primer lugar, la variable de 
confianza juega un papel preponderante en el modelo $(\beta=0.317, \mathrm{t}=4.666)$ teniendo el mayor impacto en la varianza de la variable independiente, en segundo lugar, resulta el valor percibido por el cliente $(\beta=0.234, \mathrm{t}=$ 4.226), en tercer lugar, la calidad de la interacción del personal $(\beta=0.168, \mathrm{t}=2.399)$ y finalmente la calidad de la comida $(\beta=$ $0.193, \mathrm{t}=2.378)$. El buscar entender la historia detrás de los resultados resulta interesante al usar diferentes teorías relacionadas con el comportamiento del consumidor en relación a los modelos de la salud, los cuales buscan proponer una explicación de como son diferentes las decisiones de consumo cuando existe o podría existir un efecto relacionado con la salud. Es por eso que parece pertinente el contexto de estos modelos en un contexto de pandemia mundial. De forma particular, en este estudió se propuso el Modelo de las Creencias de la Salud, el cual sugiere 3 escenarios, el primero consiste en comprender el perfil demográfico y de estilo de vida del sujeto de estudio. Este perfil refleja el bagaje en el consumidor el cual se hace notar a través de diversas creencias respecto a la salud, en este segundo escenario, la percepción juega un papel fundamental y ahí es donde hace sentido que la confianza como valor tenga una mayor importancia en un contexto de pandemia que las mismas características de la comida. Finalmente, y como tercer escenario, consecuencia de las creencias y las percepciones sobre los riesgos potenciales de la salud, se toman acciones o comportamientos, en el caso particular de esta investigación, el tomar la decisión de volver a regresar o no a un establecimiento del tipo restaurante de servicio completo.

Sin duda, una pandemia mundial con las características de la vivida en el año 2020 por el virus SARS-CoV-2, abrió la oportunidad de investigar efectos nuevos en prácticamente todos los rubros de la ciencia, al ser un evento sin precedentes. El análisis del consumidor y la hospitalidad no fueron excepciones. Resultado de este documento, se abren algunas sugerencias para poder enriquecer esta línea de investigación a través de futuros estudios: (1) considerando el Modelo de Creencias de la salud, sería muy interesante indagar los efectos de las variables de este estudio en diferentes variables demográficas, como pueden ser edad y/o género para identificar la existencia de diferencias significativas, para que en caso de existir, los restauranteros puedan crear protocolos que hagan sentido a los diferentes segmentos. (2) Puede resultar interesante el analizar bajo estas condiciones otro tipo de comportamiento como puede ser la intención de recomendación. (3) El replicar este estudio en un contexto cultural diferente al mexicano puede ser enriquecedor, pues en la fase uno del Modelo de Creencias de la salud también se estudian variables psicográficas. Se conoce que gran parte de las creencias individuales son resultado de su entorno cultural.

Finalmente, se reconoce que la principal limitante en este estudio se encontró en la metodología de muestreo, si bien se generaron preguntas filtro para llegar a los casos adecuados y se eliminaron los que parecían tener patrones repetidos, la técnica usada fue no probabilística por conveniencia, a través de una plataforma en línea. Esta decisión se tomó por la complejidad de poder levantar datos de alguna otra manera o en el campo en plena pandemia, al ser otro el contexto, se hubieran considerado otras alternativas de recolección como primera opción. 


\section{REFERENCIAS}

Adam, I., Adongo, C. A., \& Dayour, F. (2015). International tourists' satisfaction with Ghanaian upscale restaurant services and revisit intentions. Journal of Quality Assurance in Hospitality \& Tourism, 181-201.

Chen, J. V., Htaik, S., Hiele, T. M., \& Chen, C. (2017). Investigating international tourists' intention to revisit Myanmar based on need gratification, flow experience and perceived risk. Journal of Quality Assurance in Hospitality \& Tourism, 25-44.

Chen, S. C. (2012). The customer satisfaction-loyalty relation in an interactive e-service setting: The mediators. Journal of retailing and consumer services, 202-210.

Chien, C. F., Hong, T. Y., \& Guo, H. Z. (2017). An empirical study for smart production for TFTLCD to empower Industry 3.5. Journal of the Chinese Institute of Engineers, 552-561.

Freimuth, V. S., Musa, D., Hilyard, K., Quinn, S., \& Kim, K. (2014). Trust during the early stages of the 2009 H1N1 pandemic. . Journal of health communication, 321-339.

Gao, G. F. (08 de Marzo de 2018). From “A ”IV to “Z”IKV: Attacks from Emerging and Reemerging Pathogens. Obtenido de Cell: https://www.cell.com/cell/fulltext/S00928674(18)30169-

7?_returnURL=https\%3A\%2F\%2Flinkinghub.elsevier.com\%2Fretrieve\%2Fpii\%2FS00928 $67418301697 \% 3$ Fshowall\%3Dtrue

Hair Jr., J. S., Sarstedt, M., Hopkins, L., \& Kuppelwieser, V. G. (2014). Partial least squares structural equation modeling (PLS-SEM): An emerging tool in business research. European business review.

Harker, M. J., \& Egan, J. (2006). The past, present and future of relationship marketing. Journal of Marketing Management, 215-242.

Jalilvand, M. R., Salimipour, S., Elyasi, M., \& Mohammadi, M. (2017). Factors influencing word of mouth behaviour in the restaurant industry. Marketing Intelligence \& Planning.

Kim, D. J., Ferrin, D. L., \& Rao, H. R. (2008). A trust-based consumer decision-making model in electronic commerce: The role of trust, perceived risk, and their antecedents. Decision support systems, 544-564.

Ling, K. C., Chai, L. T., \& Piew, T. H. (2010). The 'Inside-out'and 'Outside-in'approaches on students' perceived service quality: An empirical evaluation. Management Science and Engineering, 1-26.

Maiman, L. A., \& Becker, M. H. (1974). The health belief model: Origins and correlates in psychological theory. Health education monographs, 336-353.

Mallery, P., \& George, D. (2000). SPSS for windows step by step. Allyn \& Bacon, Inc.

Mattila, A. S. (2001). Emotional bonding and restaurant loyalty. Cornell Hotel and Restaurant Administration Quarterly, 73-79.

Murray, D. R., \& Schaller, M. (2010). Historical prevalence of infectious diseases within 230 geopolitical regions: A tool for investigating origins of culture. Journal of Cross-Cultural Psychology, 387-409.

Namkung, Y., \& Jang, S. (2007). Does food quality really matter in restaurants? Its impact on customer satisfaction and behavioral intentions. Journal of Hospitality \& Tourism Research, 387-409.

Namkung, Y., \& Jang, S. S. (2008). Are highly satisfied restaurant customers really different? A quality perception perspective. International Journal of Contemporary Hospitality Management.

Parsa, H. G., Self, J. T., Njite, D., \& King, T. (2005). Why restaurants fail. Cornell Hotel and Restaurant Administration Quarterly, 304-322.

Rajput, A., \& Gahfoor, R. Z. (2020). Satisfaction and revisit intentions at fast food restaurants. Future Business Journal, 1-12.

Severson, K., \& Yaffe-Bellany, D. (2020). Independent restaurants brace for the unknown. The New York Times, 21. 
Sivadas, E., \& Jindal, R. P. (2017). Alternative measures of satisfaction and word of mouth. ournal of Services Marketing.

Statista. (2021). Real restaurants \& hotels consumer spending forecast in the World 2010-2025. Obtenido de Statista: https://www.statista.com/forecasts/1160448/real-restaurants-andhotels-consumer-spending-forecast-in-the-world

Sweeney, J. C., \& Soutar, G. N. (2001). Consumer perceived value: The development of a multiple item scale. . Journal of retailing, 203-220.

Vesel , P., \& Zabkar, V. (2010). Comprehension of relationship quality in the retail environment. Managing Service Quality: An International Journal.

Vesel, P., \& Zabkar, V. (2009). Managing customer loyalty through the mediating role of satisfaction in the DIY retail loyalty program. Journal of Retailing and consumer Services, 396-406.

Zeithaml, V. A., Parasuraman, A., Berry, L. L., \& Berry, L. L. (1990). Delivering quality service: Balancing customer perceptions and expectations. Simon and Schuster. 


\section{TABLAS Y FIGURAS.}

Tabla 1: Estudios empíricos realizados en un contexto restaurantero.

\begin{tabular}{|c|c|c|c|c|}
\hline Autores & Variables & Método & $\begin{array}{l}\text { Área } \\
\text { geográfica }\end{array}$ & Visitantes/Locales \\
\hline Erkmen (2019) & $\begin{array}{l}\text { Aspecto cultural de } \\
\text { la comida, servicio }\end{array}$ & Cuestionario & Turquía & Visitantes \\
\hline Vu et al. (2019) & $\begin{array}{l}\text { Servicio, precio y } \\
\text { ambiente }\end{array}$ & $\begin{array}{l}\text { Minería de textos } \\
\text { - Reseñas en } \\
\text { línea }\end{array}$ & Australia & Visitantes \\
\hline Gan et al. (2017) & $\begin{array}{l}\text { Comida, servicio, } \\
\text { ambiente, precio }\end{array}$ & $\begin{array}{l}\text { Minería de textos } \\
\text { - Reseñas en } \\
\text { línea }\end{array}$ & $E U A$ & No diferenciado \\
\hline Yan et al. (2015) & $\begin{array}{l}\text { Servicio, ambiente, } \\
\text { comida, precio y } \\
\text { calidad }\end{array}$ & $\begin{array}{l}\text { Minería de textos } \\
\text { - Reseñas en } \\
\text { línea }\end{array}$ & China & No diferenciado \\
\hline Zhang et al. (2014) & $\begin{array}{l}\text { Comida, ambiente, } \\
\text { servicio y precio }\end{array}$ & $\begin{array}{l}\text { Minería de textos } \\
\text { - Reseñas en } \\
\text { línea }\end{array}$ & China & No diferenciado \\
\hline Parsa et al. (2012) & $\begin{array}{l}\text { Comida, servicio y } \\
\text { ambiente }\end{array}$ & $\begin{array}{l}\text { Escenario } \\
\text { experimental y } \\
\text { cuestionario }\end{array}$ & EUA & No diferenciado \\
\hline Jeong y Jang (2011) & $\begin{array}{l}\text { Comida, servicio, } \\
\text { ambiente y precio }\end{array}$ & Cuestionario & EUA & No diferenciado \\
\hline Hyun (2010) & $\begin{array}{l}\text { Comida, servicio, } \\
\text { precio, ubicación y } \\
\text { ambiente }\end{array}$ & Cuestionario & EUA & No diferenciado \\
\hline Ryu y Han (2010) & $\begin{array}{l}\text { Comida, servicio y } \\
\text { ambiente }\end{array}$ & Cuestionario & EUA & No diferenciado \\
\hline Liu y Jang (2009) & $\begin{array}{l}\text { Comida, limpieza, } \\
\text { servicio y precio }\end{array}$ & Cuestionario & EUA & No diferenciado \\
\hline Namkung y Jang (2008) & $\begin{array}{l}\text { Comida, ambiente } \\
\text { y servicio }\end{array}$ & Cuestionario & EUA & No diferenciado \\
\hline
\end{tabular}

Fuente: Elaboración propia. 
Tabla 2: Items y variables del instrumento de investigación.

\begin{tabular}{|c|c|c|}
\hline $\begin{array}{l}\text { No. } \\
\text { Item }\end{array}$ & Reactivo & Variable \\
\hline 1 & ¿La presentación de la comida fue visualmente atractiva? & Calidad de la comida \\
\hline 2 & ¿El restaurante ofrece comida con buen sabor? & \\
\hline 3 & ¿El restaurante sirvió los alimentos en la temperatura adecuada? & \\
\hline 1 & $\begin{array}{l}\text { ¿Podría decir que los precios que pagué en este restaurante valen la } \\
\text { pena? }\end{array}$ & $\begin{array}{l}\text { Valor percibido por el } \\
\text { cliente }\end{array}$ \\
\hline 2 & $\begin{array}{l}\text { ¿Podría decir que la experiencia de este restaurante es de } \\
\text { extremadamente buen valor por el dinero pagado? }\end{array}$ & \\
\hline 1 & ¿Podría decir que el personal le atendió de forma excelente? & $\begin{array}{l}\text { Calidad de la } \\
\text { interacción del } \\
\text { personal }\end{array}$ \\
\hline 2 & ¿Podría decir que el personal del restaurant fue amable? & \\
\hline 3 & $\begin{array}{l}\text { ¿Podría decir que el personal del restaurante atendió de forma rápida a } \\
\text { sus necesidades? }\end{array}$ & \\
\hline 4 & ¿Se podría decir que la interacción con el personal fue apropiada? & \\
\hline 1 & $\begin{array}{l}\text { ¿El restaurante está llevando acabo las medidas de salud COVID-19 } \\
\text { recomendadas? }\end{array}$ & Confianza \\
\hline 2 & ¿El restaurante actúa pensando en los intereses de sus clientes? & \\
\hline 3 & ¿Puedo confiar en el restaurante? & \\
\hline 4 & $\begin{array}{l}\text { ¿Considera que el restaurante seguirá tomando las medidas necesarias } \\
\text { para salvaguardar la salud de sus clientes? }\end{array}$ & \\
\hline 1 & ¿Seguiré visitando este restaurante en el futuro? & Intención de revisitar \\
\hline 2 & $\begin{array}{l}\text { ¿Seguiré visitando este restaurante con al menos la misma frecuencia con } \\
\text { la que lo he hecho en el pasado? }\end{array}$ & \\
\hline
\end{tabular}


Tabla 3: Demográficos de la muestra.

\begin{tabular}{ccc} 
Género & Porcentaje & Absolutos \\
\hline Hombres & $47.99 \%$ & 155 \\
Mujeres & $52.01 \%$ & 168 \\
Total & $100.00 \%$ & 323 \\
Edad & Porcentaje & Absolutos \\
\hline menos de 20 & $2.79 \%$ & 9 \\
$21-25$ & $11.76 \%$ & 38 \\
$25-30$ & $10.22 \%$ & 33 \\
$31-35$ & $29.10 \%$ & 94 \\
$36-40$ & $13.93 \%$ & 45 \\
$41-45$ & $10.84 \%$ & 35 \\
$46-50$ & $7.74 \%$ & 25 \\
$51-55$ & $5.26 \%$ & 17 \\
$56-60$ & $4.02 \%$ & 13 \\
61-65 & $2.79 \%$ & 9 \\
66 o más & $1.55 \%$ & 5 \\
Total & $100.00 \%$ & 323 \\
& & \\
Educación & Porcentaje & Absolutos \\
\hline Sin estudios & $0.00 \%$ & 0 \\
Preparatoria & $9.91 \%$ & 32 \\
Licenciatura & $60.99 \%$ & 197 \\
Maestría & $20.74 \%$ & 67 \\
Doctorado & $8.36 \%$ & 27 \\
Total & $100 \%$ & 323
\end{tabular}

Fuente: Elaboración propia. 
Tabla 4: Resultado de Alfa de Cronbach.

\begin{tabular}{lcc} 
Dimensión & No. de items & $\alpha$ de Cronbach \\
\hline Calidad de la comida & 3 & 0.878 \\
Valor percibido por el cliente & 2 & 0.877 \\
Calidad de la interacción del personal & 4 & 0.907 \\
Confianza & 3 & 0.896 \\
Intención de revisitar & 2 & 0.700
\end{tabular}

Fuente: Elaboración propia a través de los datos de salida de SPSS. 
Tabla 5: Test de Kaiser-Meyer-Olking y prueba de esfericidad de Bartlet.

$\begin{array}{ll}\text { Kaiser-Meyer-Olkin } & 0.904 \\ \text { Test de esfericidad de Barttlet } & \\ \text { Aprox } X^{2} & 3109.44 \\ \text { Gl } & 78 \\ \text { Sig. } & 0.000\end{array}$

Fuente: Elaboración propia a través de los datos de salida de SPSS. 
Tabla 6: Comunalidades de items mediante componentes principales.

Items

¿La presentación de la comida fue visualmente atractiva?

¿El restaurante ofrece comida con buen sabor?

¿El restaurante sirvió los alimentos en la temperatura adecuada?

¿Podría decir que los precios que pagué en este restaurante valen la pena?

¿Podría decir que la experiencia de este restaurante es de extremadamente buen valor por el dinero pagado?

¿Podría decir que el personal le atendió de forma excelente?

¿Podría decir que el personal del restaurant fue amable?

¿Podría decir que el personal del restaurante atendió de forma rápida a sus necesidades?

¿Se podría decir que la interacción con el personal fue apropiada?

¿El restaurante está llevando acabo las medidas de salud COVID-19 recomendadas?

¿El restaurante actúa pensando en los intereses de sus clientes?

¿Puedo confiar en el restaurante?

¿Considera que el restaurante seguirá tomando las medidas necesarias para salvaguardar la salud de sus clientes?
Inicial Extracción

0.773

0.853

$1 \quad 0.791$

$1 \quad 0.898$

$1 \quad 0.886$

$1 \quad 0.840$

$1 \quad 0.810$

$1 \quad 0.735$

$1 \quad 0.814$

$1 \quad 0.822$

$1 \quad 0.735$

$1 \quad 0.820$

$1 \quad 0.737$

Fuente: Elaboración propia a través de los datos de salida de SPSS. 
Tabla 7: Estadística descriptiva y correlación de Pearson.

\begin{tabular}{lccccc}
\multicolumn{1}{c}{ Variable } & Media & $\begin{array}{c}\text { Desv. } \\
\text { Estandar }\end{array}$ & 1 & 2 & 3 \\
\hline 1. Calidad de la comida & 4.53 & 0.590 & & & \\
2. Valor percibido por el cliente & 4.18 & 0.829 & $.628 * *$ & & \\
3. Calidad de la interacción del personal & 4.42 & 0.647 & $.538^{* *}$ & $.546^{* *}$ & \\
4. Confianza & 4.41 & 0.677 & $.593 * *$ & $.515^{* *}$ & $.601 * *$ \\
** P-valor $<0.000$ & & & & &
\end{tabular}

Fuente: Elaboración propia a través de los datos de salida de SPSS. 
Tabla 8: Resultados de la regresión lineal múltiple.

\begin{tabular}{|c|c|c|c|c|c|c|c|c|c|c|}
\hline & $R$ & $R^{2}$ & $\begin{array}{c}R^{2} \\
\text { ajustada }\end{array}$ & $\begin{array}{c}\text { Error } \\
\text { estándar }\end{array}$ & $\begin{array}{c}\text { Cambio en } \\
R^{2}\end{array}$ & $\begin{array}{c}\text { Cambio } \\
\text { en F }\end{array}$ & gl1 & $\mathrm{g} 12$ & $\begin{array}{l}\text { Sig. Cambio } \\
\text { en F }\end{array}$ & $\begin{array}{l}\text { Durbin- } \\
\text { Watson }\end{array}$ \\
\hline & 65 & $+\mathrm{T}$ & 0.424 & 0.603 & 0.01 & 5.653 & 1 & 317 & 0.018 & 1.9 \\
\hline
\end{tabular}

Fuente: Elaboración propia a través de los datos de salida de SPSS. 
Tabla 9: Resultados del modelo ANOVA

\begin{tabular}{lccccc} 
& Suma de & \multicolumn{1}{c}{ Media } \\
Modelo 1 & cuadrados & gl & cuadrática & F & Sig \\
\hline Regresión & 87.649 & 4 & 21.912 & 60.183 & 0.000 \\
Residuo & 115.418 & 317 & 0.364 & & \\
Total & 203.067 & 321 & & &
\end{tabular}

Fuente: Elaboración propia a través de los datos de salida de SPSS. 
Tabla 10: Coeficientes de la regresión.

\begin{tabular}{|c|c|c|c|c|c|c|c|}
\hline \multirow[b]{2}{*}{ Modelo 1} & \multicolumn{2}{|c|}{$\begin{array}{l}\text { Coeficientes no } \\
\text { estandarizados }\end{array}$} & \multirow{2}{*}{$\begin{array}{c}\text { Coeficientes } \\
\text { estandarizados } \\
\text { B } \\
\end{array}$} & \multirow[b]{2}{*}{$\mathrm{t}$} & \multirow[b]{2}{*}{ Sig. } & \multicolumn{2}{|c|}{ Estadísticas de colinealidad } \\
\hline & B & Desv. Error & & & & Tolerancia & VIF \\
\hline Constante & 0.233 & 0.287 & & 0.810 & & & \\
\hline TST & 0.317 & 0.068 & 0.271 & 4.666 & 0.000 & 0.532 & 1.881 \\
\hline VPC & 0.234 & 0.055 & 0.244 & 4.226 & 0.000 & 0.538 & 1.858 \\
\hline CIP & 0.168 & 0.070 & 0.137 & 2.399 & 0.017 & 0.551 & 1.815 \\
\hline CQ & 0.193 & 0.081 & 0.143 & 2.378 & 0.018 & 0.495 & 2.018 \\
\hline
\end{tabular}

Fuente: Elaboración propia a través de los datos de salida de SPSS. 
Tabla 11: Contraste de hipótesis de investigación.

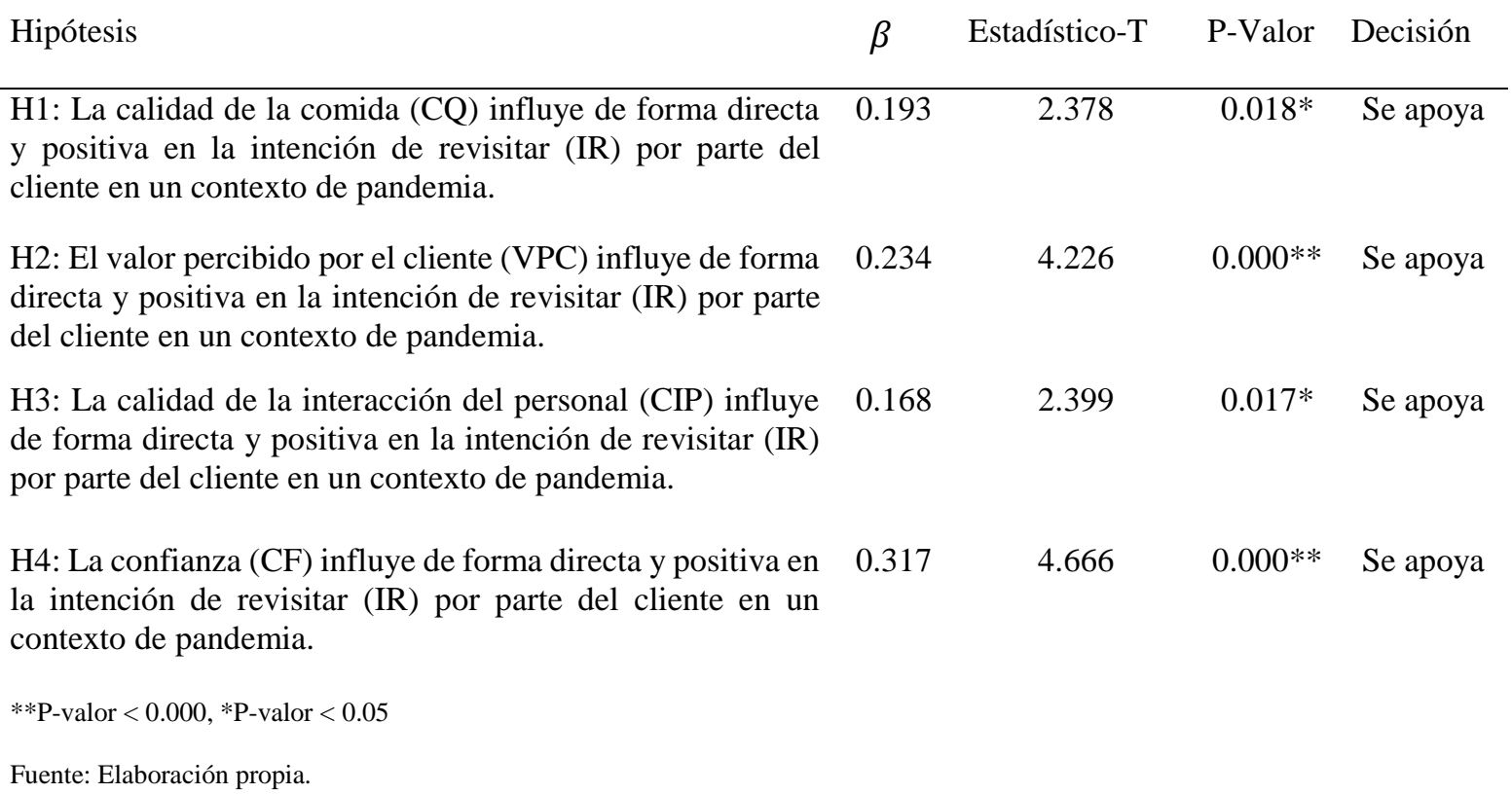


Figura 1: Pronóstico del consumo real en restaurantes alrededor del mundo, desde 2010 hasta el 2025 (en Millones de dóleres).

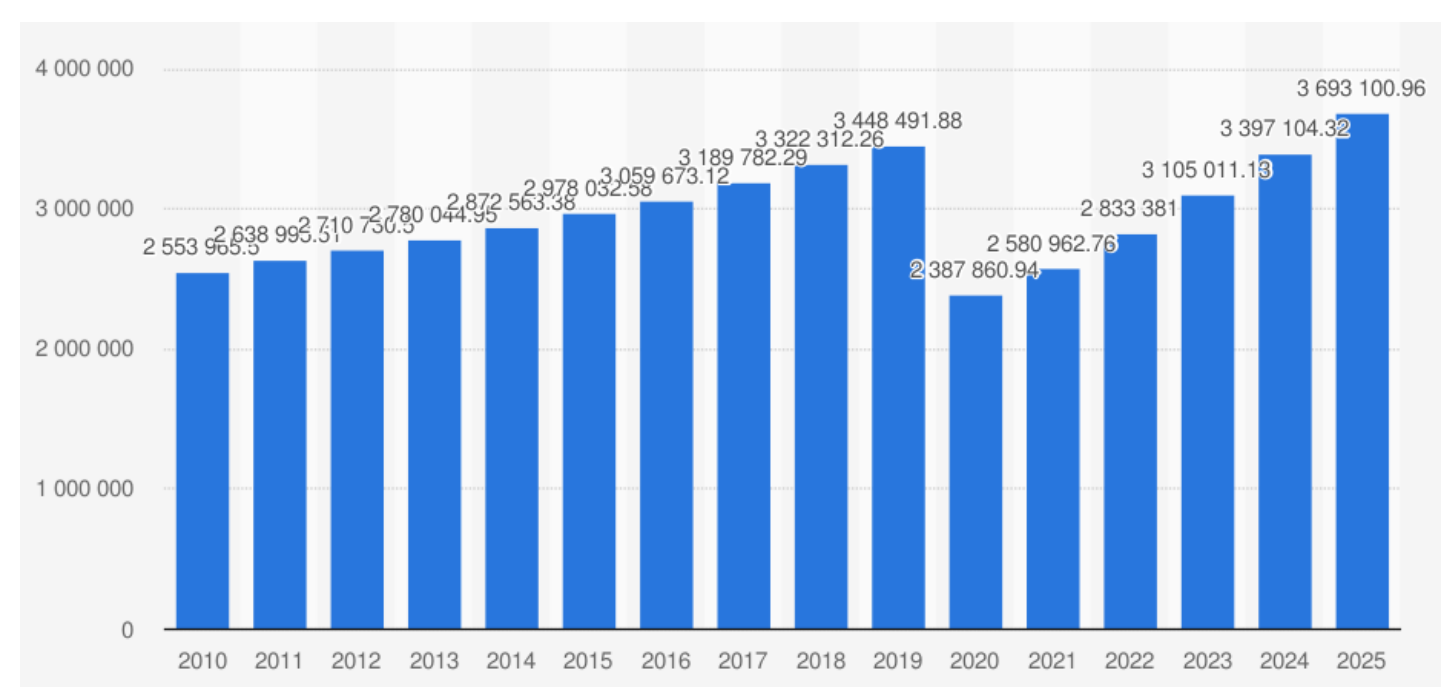

Fuente: Statista 2021. 
Figura 2: Modelo gráfico de la investigación.

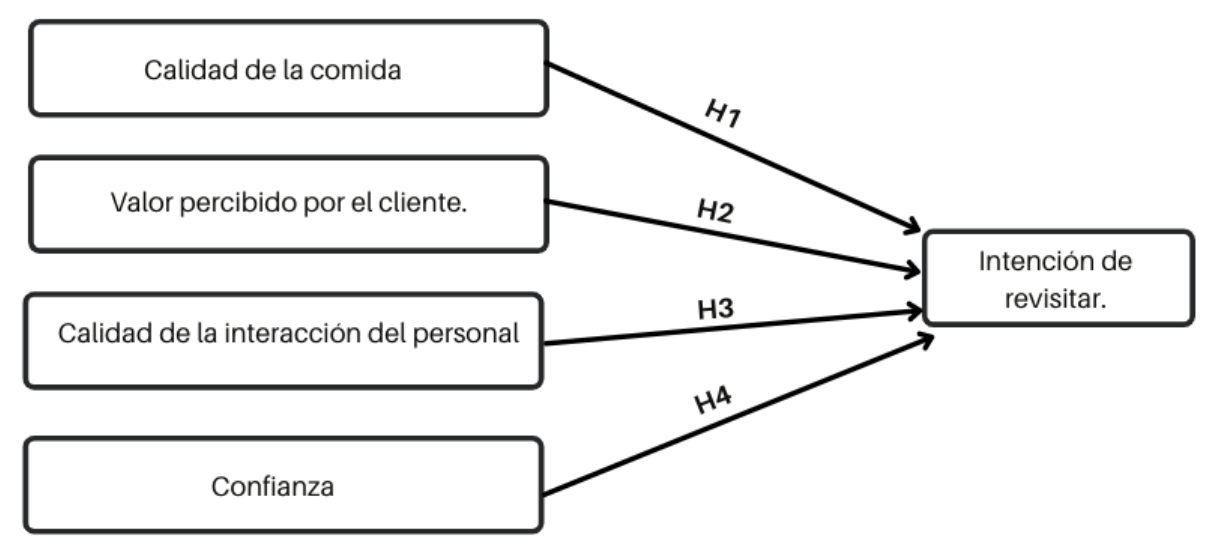

Fuente: Elaboración propia. 
Figura 3: Escala likert de 5 puntos en formato smiley.

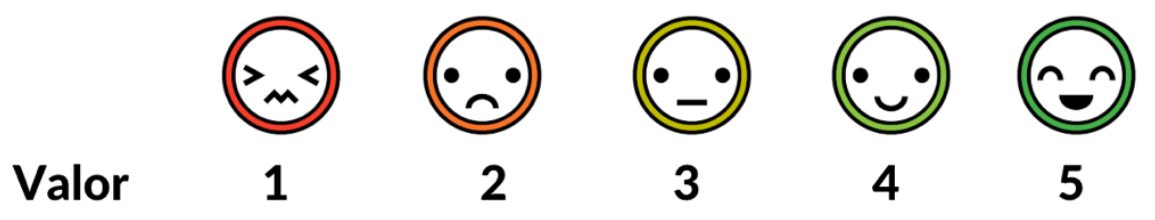

Fuente: Obtenido de la plataforma Kindorse surveys. 